\title{
Correlation between salivary cortisol levels and Hospital Anxiety and Depression scores in oral lichen planus and recurrent aphthous stomatitis
}

\author{
Vaishnavee Vassandacoumara', Jonathan M. Daniel ${ }^{2}$ \\ ${ }^{1}$ Department of Oral Medicine and Radiology, K. M. Shah Dental College and Hospital, Piparia, Vadodara 391760, India. \\ ${ }^{2}$ Department of Oral Medicine and Radiology, Mahatma Gandhi Postgraduate Institute of Dental Sciences, Puducherry 605006, India
}

Correspondence to: Dr. Vaishnavee Vassandacoumara, Department of Oral Medicine and Radiology, K. M. Shah Dental College and Hospital, Piparia, Vadodara 391760, India. E-mail: vaish.ajith@gmail.com

How to cite this article: Vassandacoumara V, Daniel JM. Correlation between salivary cortisol levels and Hospital Anxiety and Depression scores in oral lichen planus and recurrent aphthous stomatitis. Stomatological Dis Sci 2017;1:103-8.

\section{Article history: \\ Key words: \\ Anxiety, \\ depression, \\ hydrocortisone, \\ lichen planus, \\ oral, \\ stomatitis, \\ aphthous, \\ saliva}

Received: 25 May 2017

First Decision: 27 Jul 2017

Revised: 13 Aug 2017

Accepted: 26 Oct 2017

Published: 25 Dec 2017

\begin{abstract}
Aim: The aim of this study was to estimate the salivary cortisol levels in patients with oral lichen planus (OLP) and recurrent aphthous stomatitis (RAS) and to correlate it with their psychological profile using the Hospital Anxiety and Depression (HAD) scale. Methods: The study population comprised of 60 patients - 20 with OLP, 20 with RAS and 20 healthy controls. Salivary cortisol levels were assessed using the chemiluminescent immuno assay technique. Anxiety and depression levels were evaluated using the HAD scale. Results: The Kruskal Wallis mean ranks of salivary cortisol level in the OLP and RAS groups were 33.45 and 33.48 respectively and were higher than the mean rank of the control group with 24.58. But this difference was not statistically significant $(P=0.178)$. The mean ranks of anxiety score in the OLP and RAS groups were 36.48 and 39.50. The mean ranks of depression scores were 41.88 and 35.25 in the OLP and RAS groups respectively. The control group had a statistically significant lower mean ranks for anxiety and depression $(P<0.001)$. Anxiety and depression scores showed strong positive correlation in both patient groups. Conclusion: The results suggest that anxiety and depression play a role in the pathogenesis of OLP and RAS. The role of salivary cortisol as a biomarker of stress remains to be validated. Patients suffering from psychosomatic diseases such as OLP and RAS need a comprehensive and holistic treatment approach to manage effectively the psychosocial factors involved in the disease process.
\end{abstract}

\section{INTRODUCTION}

Oral changes with psychosomatic aetiology are still an insufficiently confirmed and investigated subgroup of psychosomatic diseases which have long been known in medicine. Many of them, which are believed to be of psychosomatic character, still do not have sufficient explanation of their aetiology, or it is considered to be multicausal ${ }^{[1]}$.

Evidence supports the idea that stress and psychologic

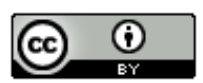

This is an open access article licensed under the terms of Creative Commons Attribution 4.0 International License (https://creativecommons.org/licenses/by/4.0/), which permits unrestricted use, distribution, and reproduction in any medium, as long as the original author is credited and the new creations are licensed under the identical terms.

For reprints contact: service@oaepublish.com

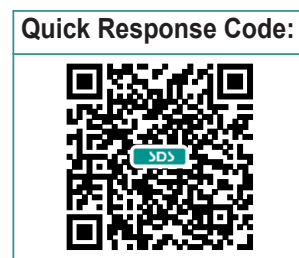


illness can modify immunological functions ${ }^{[2]}$. Individuals exposed to experimental stress situations respond with increased secretion of cortisol ${ }^{[3]}$. Salivary cortisol measurements are considered to be an excellent indicator of plasma free cortisol concentrations. They escape the biological changes due to corticosteroidbinding globulin alterations and provide a feasible approach to assess pituitary adrenal function ${ }^{[4]}$. The serum is the gold standard of diagnostic media; however, there are circumstances where saliva has a decided advantage over the serum. The collection of saliva is relatively safe, non-invasive, and simple and it may repeatedly be collected without causing discomfort to the patient. It is inexpensive, easy to perform and avoids a probable rise in levels due to the apprehension of venepuncture.

Oral lichen planus (OLP) is a T cell-mediated autoimmune disease in which autocytotoxic $\mathrm{CD} 8^{+} \mathrm{T}$ cells trigger apoptosis of oral epithelial cells ${ }^{[5]}$. The waxing and waning nature of the lesions have been attributed to the perception of stress ${ }^{[6]}$. Several studies have reported a relationship between recurrent aphthous stomatitis (RAS) and various causes, but the results are conflicting ${ }^{[7]}$. Stress has also been postulated as a precipitating factor in RAS ${ }^{[8]}$.

The Hospital Anxiety and Depression (HAD) scale measures anxiety and depression separately on a 21 point scale. It comprises of 14 questions which are answered by the patients; 7 questions for anxiety and 7 questions for depression with scoring for each question ranging from 0 to 3 . Lamey and Lamb $^{[9]}$ in 1989 reported that the HAD scale is easy to understand and quick to complete. They also compared the HAD scale with the MontgomeryAshberg depression rating scale and the irritabilitydepression-anxiety scale, thereby validating it. The authors concluded that the HAD scale could perform the function of a screening device for the presence or absence of clinical mood disorder as well as provide an ongoing record of the patient's progress at subsequent visits to the clinic $^{[9]}$.

With this background, this study was taken up to determine the salivary cortisol levels and the psychosocial factors using the HAD scores in patients with OLP and RAS. The null hypothesis stated that there is no difference in the salivary cortisol levels and HAD scores in OLP and RAS patients as compared with healthy non-diseased individuals.

\section{METHODS}

A single-center cross-sectional study was conducted in the Department of Oral Medicine and Radiology at Mahatma Gandhi Postgraduate Institute of Dental Sciences, Puducherry, India. The study was reviewed and approved by the Ethics and Research Committee of the Institution. Written informed consent was obtained from all individual participants included in the study. We have studied two stress-related oral diseases, OLP and RAS.

Twenty consecutive patients who were clinically diagnosed with OLP were further advised for histopathological confirmation for OLP. They comprised of one patient group. Twenty patients clinically diagnosed with minor RAS as per Natah et al. ${ }^{[10]}$ formed the other patient group. Twenty healthy volunteers who reported for routine dental examination were included as controls. Patients with systemic diseases such as diabetes or hypertension or those with history of recent or on current steroid therapy were excluded from the study. Patients with known adrenal hypersecretion or hyposecretion, pregnant patients or tobacco users were excluded from the study. Patients who were not willing to give the written informed consent were not enrolled in the study.

All the study participants were asked to complete the HAD questionnaire with regard to how they felt over the previous 1 week. The questionnaire was translated into the vernacular language and validated. The interview method was followed to complete the questionnaire from illiterate patients. The interviewer was blinded to the study groups. Scores of 0-7 in respective anxiety and depression subscales are considered normal, with 8-10 borderline and 11-21 indicating clinical "caseness".

An unstimulated whole saliva sample was collected from the patients as well as the controls by the spit method in sterile plastic containers. To counter the effect of diurnal variations in salivary cortisol, the collection of salivary samples were performed between 8:00 and 9:00 am. All samples were deep frozen for at least $3 \mathrm{~h}$ or overnight. Then the samples were thawed again, mixed and centrifuged for $5 \mathrm{~min}$, in order to obtain a clear supernatant. If the supernatant was still not completely clear, the freeze/ thaw cycle was repeated as many times as needed and the centrifugation time was extended to $15 \mathrm{~min}$. Then the clear supernatant is visually inspected in front of a white background. Any sample with slightly red color was discarded.

Salivary cortisol was determined by using the chemiluminescent immuno assay technique using the ADVIA $^{\circledR}$ Centaur $^{\mathrm{TM}}$ System and ADVIA $^{\circledR}$ 
Table 1: Gender distribution of the study groups, $n$ (\%)

\begin{tabular}{|c|c|c|c|}
\hline \multirow{2}{*}{ Group } & \multicolumn{2}{|c|}{ Gender } & \multirow{2}{*}{ Total } \\
\hline & Male & Female & \\
\hline OLP & $13(65)$ & 7 (35) & $20(100)$ \\
\hline RAS & $10(50)$ & $10(50)$ & 20 (100) \\
\hline Control & $10(50)$ & $10(50)$ & 20 (100) \\
\hline Total & $33(55)$ & $27(45)$ & 60 (100) \\
\hline
\end{tabular}

OLP: oral lichen planus; RAS: recurrent aphthous stomatitis

Centaur ${ }^{\mathrm{TM}}$ Cortisol Lite Reagent and Solid Phase. The ADVIA Centaur Cortisol assay measures cortisol concentrations up to $75 \mu \mathrm{g} / \mathrm{dL}(2,069 \mathrm{nmol} / \mathrm{L})$ with a minimum detectable concentration (analytical sensitivity) of $0.20 \mu \mathrm{g} / \mathrm{dL}(5.5 \mathrm{nmol} / \mathrm{L})$.

\section{RESULTS}

The salivary cortisol levels of the patient and control groups were assessed and recorded. The HAD scale scores were calculated and recorded as anxiety subscale scores and depression subscale scores. The Kruskal Wallis test was used to statistically analyze the mean ranks between the study groups. Spearmann's correlation coefficient was applied to the study parameters to measure the correlation among these variables for all the study groups. The $P$ value was set at 0.05 or less for statistical significance.

The mean ages of the OLP group and RAS group were 42.31 and 33.50 years respectively. The mean age of the control group was 34.10 years. In the OLP group, $65 \%$ of patients were males. In the RAS group, the male:female ratio was 1:1 [Table 1].

The mean salivary cortisol levels in the OLP group and the RAS group were $0.83 \pm 0.40$ and $0.82 \pm 0.38 \mu \mathrm{g} / \mathrm{dL}$ respectively, which were higher than the control group with a mean salivary cortisol of $0.64 \pm 0.33 \mu \mathrm{g} / \mathrm{dL}$. The mean anxiety score was highest in the RAS group with $9.25 \pm 4.04$ followed by the OLP group with $8.50 \pm 3.59$. The mean depression score was highest in the OLP group with $8.05 \pm 3.93$ followed by the RAS group with $6.45 \pm 3.93$. The mean anxiety and mean depression scores of the control group were $3.55 \pm 1.54$ and 1.25 \pm 1.80 respectively [Table 2 ].

The proportion of patients with borderline or clinically morbid anxiety was $87.50 \%$ and $93.75 \%$ in the groups of OLP and RAS respectively. The proportion of patients with borderline or clinically morbid depression was $87.50 \%$ and $56.25 \%$ in the groups of OLP and RAS respectively.

The "Kruskal Wallis test" was applied to the salivary cortisol levels and anxiety and depression scores
Table 2: Mean salivary cortisol levels, anxiety scores and depression scores between study groups

\begin{tabular}{lcccc}
\hline Group & Variables & Number & Mean & SD \\
\hline OLP & Cortisol & 20 & 0.8265 & 0.4017 \\
& Anxiety & 20 & 8.50 & 3.591 \\
\multirow{4}{*}{ RAS } & Depression & 20 & 8.05 & 3.927 \\
& Cortisol & 20 & 0.8180 & 0.3798 \\
& Anxiety & 20 & 9.25 & 4.038 \\
Control & Depression & 20 & 6.45 & 3.927 \\
& Cortisol & 20 & 0.6435 & 0.3269 \\
& Anxiety & 20 & 3.55 & 1.538 \\
& Depression & 20 & 1.25 & 1.803 \\
\hline
\end{tabular}

OLP: oral lichen planus; RAS: recurrent aphthous stomatitis

to measure the difference in mean ranks between patient groups and control group as shown in Table 3. The mean ranks of salivary cortisol level in the OLP and RAS groups were 33.45 and 33.48 respectively and were higher than the mean rank of the control group with 24.58. But this difference was not statistically significant $(P=0.178)$. The mean ranks of anxiety score in the OLP and RAS groups were 36.48 and 39.50 . The mean ranks of depression scores were 41.88 and 35.25 in the OLP and RAS groups respectively. The control group had a statistically significant lower mean ranks of anxiety and depression $(P<0.001)$.

Spearmann's correlation coefficient was employed to estimate the correlation among the variables for all the study groups. A significant strong positive correlation between anxiety and depression scores was seen in the OLP and RAS groups. Anxiety scores and salivary cortisol levels; and depression scores and salivary cortisol levels were not positively correlated in any of the study groups [Tables 4-6].

\section{DISCUSSION}

Research on oral psychosomatic diseases has not yielded conclusive results. The etiopathogenesis of OLP appears to be complex interactions with genetic, environmental and lifestyle factors ${ }^{[11]}$. Stress has been widely held to be an important etiological factor in OLP and some investigators have studied this disease in relation to stress with conflicting results ${ }^{[12-16]}$. Allen et al. ${ }^{[12]}$ suggested that patients who manifest OLP have no greater tendency toward anxiety and no more stressful life events than other individuals. Koray et al. ${ }^{[11]}$ concluded that OLP is closely related to stress and that besides traditional treatment, psychological support is also needed. Researchers have found fine differences in psycho-immune interactions between patients afflicted with nonerosive OLP lesions compared to those with erosive OLP lesions ${ }^{[13]}$. Rödström et al. ${ }^{[16]}$ reported that there 
Table 3: Kruskal Wallis mean ranks between groups

\begin{tabular}{lccccccc}
\hline \multirow{5}{*}{ Cortisol } & Group & Number & $\begin{array}{c}\text { Mean } \\
\text { rank }\end{array}$ & $\begin{array}{c}\text { Chi- } \\
\text { square }\end{array}$ & df & $\boldsymbol{P}$-value \\
& OLP & 20 & 33.45 & 3.454 & 2 & 0.178 \\
& RAS & 20 & 33.48 & & & \\
Anxiety & Control & 20 & 24.58 & & & \\
& OLP & 20 & 36.48 & 22.543 & 2 & $<0.001$ \\
& RAS & 20 & 39.50 & & & \\
\multirow{5}{*}{ Depression } & Control & 20 & 15.52 & & & \\
& OLP & 20 & 41.88 & 27.400 & 2 & $<0.001$ \\
& RAS & 20 & 35.25 & & & \\
& Control & 20 & 14.38 & & & \\
\hline
\end{tabular}

OLP: oral lichen planus; RAS: recurrent aphthous stomatitis

Table 4: Correlation coefficient for the OLP group

\begin{tabular}{lccc}
\hline Variables & Statistics & Anxiety & Depression \\
\hline Cortisol & Correlation coefficient & -0.188 & -0.161 \\
& $P$-value & 0.427 & 0.498 \\
Anxiety & Correlation coefficient & & 0.815 \\
& $P$-value & & $<0.001$ \\
\hline
\end{tabular}

OLP: oral lichen planus

was no support for an impaired capacity of OLP patients to suppress an immune response through cortisol induction in conjunction with experimental stress.

Stress and anxiety may play a significant role in the onset and recurrence of RAS lesions ${ }^{[6]}$. RAS represents a very common but poorly understood mucosal disorder. It affects men and women of all races, ages and geographic regions. At least 1 in 5 individuals have been afflicted with aphthous ulcers at least once in their lifetime. Although different aetiologies and mechanisms might be operative in the aetiopathogenesis of aphthous ulceration; pain, recurrence, self-limitation of the condition, and destruction of the epithelium seem to be the ultimate outcomes. There is no curative therapy to prevent the recurrence of ulcers, and all available treatment modalities are directed only towards reducing the frequency or severity of the lesions ${ }^{[10]}$. The prodromal phase for RAS, recognized by most patients, consists of a burning sensation a few days before the onset of ulceration, as well as the perception of stress ${ }^{[17]}$.

Koray et al. ${ }^{[11]}$ and Lopez-Jornet et al. ${ }^{[18]}$ collected the unstimulated whole saliva sample from their study population unlike other researchers ${ }^{[7,15,16,19]}$ who collected stimulated saliva samples for their study. We have collected unstimulated whole saliva samples from our sample population despite the risk that cortisol can contaminate with gingival crevicular fluid, but the study groups had the same circumstances. Similar to earlier studies ${ }^{[8,9,15,18]}$, we have made use of the HAD scale for assessing the psychological profile
Table 5: Correlation coefficient for the RAS group

\begin{tabular}{lccc}
\hline Variables & Statistics & Anxiety & Depression \\
\hline Cortisol & Correlation coefficient & -0.043 & -0.049 \\
& $P$-value & 0.858 & 0.838 \\
Anxiety & Number & 20 & 20 \\
& Correlation coefficient & & 0.684 \\
& $P$-value & & 0.001 \\
& Number & & 20 \\
\hline
\end{tabular}

RAS: recurrent aphthous stomatitis

Table 6: Correlation coefficient for the control group

\begin{tabular}{lccc}
\hline Variables & Statistics & Anxiety & Depression \\
\hline Cortisol & Correlation coefficient & 0.204 & 0.386 \\
& $P$-value & 0.389 & 0.092 \\
Anxiety & Number & 20 & 20 \\
& Correlation coefficient & & 0.417 \\
& $P$-value & & 0.067 \\
& Number & & 20 \\
\hline
\end{tabular}

in our sample population.

\section{Salivary cortisol}

The mean salivary cortisol level in our group of OLP patients amounted to $0.83 \pm 0.40 \mu \mathrm{g} / \mathrm{dL}$ which was higher than the mean of the control group, in concordance with many earlier studies ${ }^{[11,16,18-20]}$, but the absolute value is lower than that reported in few of these studies ${ }^{[11,16,19,20]}$. This higher mean cortisol level could be attributed to the earlier time of saliva collection in our study as compared to other studies. This elevated cortisol level is supported by Pippi et al..$^{[21]}$ who reported a reduced capability of coping with stress events and impairment of hypothalamic-pituitaryadrenal axis activity with hypocortisolism detected in the morning hours in OLP patients. The mean rank of salivary cortisol in OLP patients was not statistically significantly higher than the control group. This insignificance is in concordance with Girardi et al. ${ }^{[22]}$ but dissimilar to other studies ${ }^{[11,16,18,19,20]}$.

McCartan et al. ${ }^{[8]}$ reported a statistically significant elevation of median salivary cortisol levels in their study group with persistent aphthae as compared to the other group who had been relieved of their aphthae following correction of detected haematinic deficiency states. This was similar to the results of Nadendla et al. ${ }^{[23]}$, but the difference was not statistically significant in our study.

From our study results, we found that the mean salivary cortisol level in RAS patients was $0.82 \pm$ $0.38 \mu \mathrm{g} / \mathrm{dL}$, which is lower than the mean salivary cortisol level reported by Albanidou-Farmaki et al. ${ }^{[7]}$ and Nadendla et al. ${ }^{[23]}$. The variations may be attributed to the difference in the perspective of the 
psychobiologic response to stress in these disease processes.

\section{HAD scores}

There were $87.5 \%$ of our OLP patients which had borderline or clinically morbid anxiety, as compared to the lesser proportion of $50 \%$ reported by McCartan ${ }^{[15]}$ in his OLP patients. In the study above, the proportion of patients with borderline or clinically morbid depression was only $12 \%$, in contrast to the $87.5 \%$ of our OLP patients who were depressed. Our study results suggest high levels of anxiety in OLP patients. This confirms the findings of several previous studies $^{[15,18,20,24]}$, but contradicts that of Allen et al. ${ }^{[12]}$, Pippi et al. ${ }^{[21]}$ and Girardi et al. ${ }^{[22]}$. High levels of depression in our OLP patients contradict the findings of McCartan ${ }^{[15]}$, Pippi et al. ${ }^{[21]}$ and Girardi et al. ${ }^{[22]}$, but is supported by the results of Shah et al. ${ }^{[19]}$.

The proportion of patients with borderline or clinically morbid anxiety in our RAS group was $93.75 \%$. This was higher than the $83 \%$ reported in the RAS patients of McCartan et al. ${ }^{[8]}$ Nadendla et al. ${ }^{[23]}$ reported significantly increased anxiety scores in their RAS group similar to our study results.

Interestingly, in our study, though the salivary cortisol levels of the OLP group were similar to that of the RAS group, the anxiety score of this group was lower than the RAS group. This finding is supported by Chiappelli et al. ${ }^{[6]}$ who documented that the psychologic symptoms of OLP patients are not detected by the HAD scale, whereas these patients scored high on the Hamilton anxiety scale, the Hamilton depressive scale and the profile of moods scale $^{[25]}$.

A significant positive correlation existed between anxiety and depression in the OLP group and the RAS group. In agreement with Koray et al. ${ }^{[11]}$, we, hence cannot declare whether the disease symptoms in these conditions are more related to anxiety or depression.

McCartan et al. ${ }^{[8]}$ and Shah et al. ${ }^{[19]}$ reported that anxiety scores and salivary cortisol levels were not well correlated in their RAS and OLP groups respectively. This is in concordance with our study results, wherein anxiety and salivary cortisol levels were not well correlated in both the OLP and RAS groups. We also believe that the overall elevated anxiety scores and the overall elevated salivary cortisol levels are more important than a direct comparison between the two. In contrast to our study, Nadendla et al. ${ }^{[23]}$ reported a positive correlation between salivary cortisol and anxiety in their
RAS group, and Shah et al. ${ }^{[19]}$ reported a positive correlation between salivary cortisol and depression in their OLP group.

This study has confirmed that the two most common aspects of psychosis - anxiety and depression are involved in the etiology of OLP and RAS. It is hence, recommended that treatment of patients with psychogenic aspects to their oral disease should include a psychological input as well as a biological one.

This field of research requires further study to determine whether there is a "cause" or "effect" relationship between the psychosocial factors and the psychosomatic diseases affecting the oral cavity. Future research should also be directed towards testing the role of stress in the maintenance of symptoms, response to treatment and disease progression.

Of all the determinations that can be performed in saliva, cortisol determination can exactly and efficiently quantify the biologically active cortisol. Therefore, cortisol determination constitutes an adequate method for evaluating the response to stress in humans ${ }^{[26]}$; which, when linked to the minimal invasiveness of this technique could favor its substitution of the plasmatic technique in the future.

Research to date suggests a psychosomatic component to the etiology and prognosis of OLP and RAS. These conditions are often referred to as "stress-related" or "stress-associated" diseases. Patients with these stress-related oral diseases experience significant discomfort which can adversely affect their quality of life. However, this field of stress research is highly complex and has not been studied extensively about oral changes.

The estimation of salivary cortisol can prove to be an effective physiological testing instrument for patients with oral psychosomatic diseases. It reflects individual response to stress and hence, can give us an insight into the etiopathogenesis and prognosis in these patients.

In conclusion, we suggest that oral physicians can improve the quality of care provided to patients with oral psychosomatic disease by identifying the psychosocial risk factors affecting them and that these patients may need supportive psychological management to increase their ability to cope with stress along with the conventional treatment methods. Stress management interventions such as psychological counseling, relaxation, imagery and 
meditation may benefit the patients by providing tools and methods for self-preservation and adaptation to stressful challenges.

\section{DECLARATIONS}

\section{Acknowledgments}

The authors gratefully acknowledge the statistical inputs from Dr. Pulkit Kalyan, Dr. Medha Kalyan and Dr. Sweta Singh; and also acknowledge the efforts of Dr. Jon Wanger in refining the final manuscript.

\section{Authors' contributions}

Drafted, analyzed and interpreted the data, wrote the manuscript: V. Vassandacoumara

Concept and design of the study, critically reviewed the manuscript: J.M. Daniel

\section{Financial support and sponsorship None.}

\section{Conflicts of interest}

The authors declare that they have no competing interests.

\section{Patient consent}

Written informed consent was obtained from all individual participants included in the study.

\section{Ethics approval}

The study was reviewed and approved by the Ethics and Research Committee of the Institution. All procedures performed in the study were in accordance with the ethical standards of the institutional Ethics and Research committee and with the 1964 Helsinki declaration and its later amendments or comparable ethical standards.

\section{REFERENCES}

1. Richter I, Vidas I, Tureinovie P. Relationship of psychological characteristics and oral diseases with possible psychosomatic aetiology. Acta Stomatol Croat 2003;37:35-9.

2. Jankovic BD. Neuroimmune interactions: experimental and clinical strategies. Immunol Lett 1987;16:341-53.

3. Jones DA, Rollman GB, Brooke RI. The cortisol response to psychological stress in temporomandibular dysfunction. Pain 1997;72:171-82.

4. Laudat MH, Cerdas S, Fournier C, Guiban D, Guilhaume B, Luton JP. Salivary cortisol measurement: a practical approach to assess pituitary-adrenal function. J Clin Endocrinol Metab 1988;66:343-8.

5. Eisen D, Carrozzo M, Bagan Sebastian JV, Thongprasom K. Number V Oral lichen planus: clinical features and management. Oral Dis 2005;11:338-49.

6. Chiappelli F, Cajulis Olivia S. Psychobiologic views on stress-related oral ulcers. Quintessence Int 2004;35:223-7.
7. Albanidou-Farmaki E, Poulopoulos AK, Epivatianos A, Farmakis $\mathrm{K}$, Karamouzis M, Antoniades D. Increased anxiety level and high salivary and serum cortisol concentrations in patients with recurrent aphthous stomatitis. Tohoku J Exp Med 2008;214:291-6.

8. McCartan BE, Lamey PJ, Wallace AM. Salivary cortisol and anxiety in recurrent aphthous stomatitis. J Oral Pathol Med 1996;25:357-9.

9. Lamey PJ, Lamb AB. The usefulness of the HAD scale in assessing anxiety and depression in patients with burning mouth syndrome. Oral Surg Oral Med Oral Pathol 1989;67:390-2.

10. Natah SS, Konttinen YT, Enattah NS, Ashammakhi N, Sharkey KA, Häyrinen-Immonen R. Recurrent aphthous ulcers today: a review of growing knowledge. Int J Oral Maxillofac Surg 2004;33:221-34.

11. Koray M, Dülger O, Ak G, Horasanli S, Uçok A, Tanyeri H, Badur S. The evaluation of anxiety and salivary cortisol levels in patients with oral lichen planus. Oral Dis 2003;9:298-301.

12. Allen CM, Beck FM, Rossie KM, Kaul TJ. Relation of stress and anxiety to oral lichen planus. Oral Surg Oral Med Oral Pathol 1986;61:44-6.

13. Chiappelli F, Kung MA, Nguyen P, Villanueva P, Farhadian EA, Eversole LR. Cellular immune correlates of clinical severity in oral lichen planus: preliminary association with mood states. Oral Dis 1997;3:64-72.

14. Macleod RI. Psychological factors in oral lichen planus. Br Dent $J$ 1992;173:88.

15. McCartan BE. Psychological factors associated with oral lichen planus. J Oral Pathol Med 1995;24:273-5.

16. Rödström PO, Jontell M, Hakeberg M, Berggren U, Lindstedt G. Erosive oral lichen planus and salivary cortisol. J Oral Pathol Med 2001;30:257-63

17. Chiappelli F, Abanomy A, Hodgson D, Mazey KA, Messadi DV, Mito RS, Nishimura I, Spigleman I. Clinical, experimental and translational psychoneuroimmunology research models in oral biology and medicine. In: Ader R, Cohen R, Felten D, editors. Psychoneuroimmunology. New York: Academic Press; 2001. p. 645-70.

18. Lopez-Jornet P, Cayuela CA, Tvarijonaviciute A, Parra-Perez F, Escribano D, Ceron J Oral lichen planus: salivary biomarkers, cortisol, immunoglobulin A, adiponectin. J Oral Pathol Med 2016;45:211-7.

19. Shah B, Ashok L, Sujatha GP. Evaluation of salivary cortisol and psychological factors in patients with oral lichen planus. Indian J Dent Res 2009;20:288-92.

20. Nadendla LK, Meduri V, Paramkusam G, Pachava KR. Association of salivary cortisol and anxiety levels in lichen planus patients. $J$ Clin Diag Res 2014;8:ZC01-3.

21. Pippi R, Patini R, Ghiciuc CM, Sandu RB, Pasquali V, Scaccianoce S, Dima-Cozma LC, Patacchioli FR. Diurnal trajectories of salivary cortisol, salivary $\alpha$-amylase and psychological profiles in oral lichen planus patients. $J$ Biol Regul Homeost Agents 2014;28:147-54.

22. Girardi C, Luz C, Cherubini K, de Figueiredo MA, Nunes ML, Salum FG. Salivary cortisol and dehydroepiandrosterone (DHEA) levels, psychological factors in patients with oral lichen planus. Arch Oral Biol 2011;56:864-8

23. Nadendla LK, Meduri V, Paramkusam G, Pachava KR. Relationship of salivary cortisol and anxiety in recurrent aphthous stomatitis. Indian J Endocrinol Metab 2015;19:56-9.

24. Kovesi G, Banoczy J. Follow-up studies in oral lichen planus. Int $J$ Oral Surg 1973;2:13-9.

25. Prolo P, Chiappelli F, Cajulis E, Bauer J, Spackman S, Romeo H, Carrozzo M, Gandolfo S, Christensen R. Psychoneuro-immunology in oral biology and medicine: the model of oral lichen planus. Ann $N$ Y Acad Sci 2002;220:290-7.

26. Mirasoli M, Deo SK, Lewis JC, Roda A, Daunert S. Bioluminescence immunoassay for cortisol using recombinant aequorin as a label. Anal functioning and depression: a meditation analysis. Psychiatry Res 2004;126:197-201. 